\title{
A chemical genetic approach to probe the function of PINK1 in regulating mitochondrial dynamics
}

Cell Research (2015) 25:394-397. doi:10.1038/cr.2014.159; published online 5 December 2014

\section{Dear Editor,}

PINK1 and Parkin have been implicated in mitochondrial quality control, mitochondrial fusion/fission dynamics and axonal mitochondrial mobility in neurons $[1,2]$. The PINK1-Parkin pathway is very dynamic and the biological responses mediated by the PINK1-Parkin pathway differ spatially and kinetically in different cell types [2]. Therefore, tools to dynamically probe the function of the PINK1-Parkin pathway are highly desirable. Here we report a chemical genetic approach to probe the function of PINK1 in mitophagy and mitochondrial mobility. Our results validate the roles of PINK1 in recruitment of Parkin to mitochondria, mitochondrial mobility in neurons, and mitochondrial fusion/fission dynamics. In addition, we reveal that PINK1 is required for triggering Parkin translocation to mitochondria, but is dispensable for sustaining Parkin accumulation and consequent mitophagy.

Chemical genetic approaches have recently emerged as general tools for perturbing biological processes as small-molecule perturbants often act quickly and reversibly to inhibit or activate a pathway. Shokat and colleagues [3] have demonstrated that a subset of protein kinases can be engineered to be susceptible to inhibition by protein phosphatase 1 (PP1) analogs, whereas few, if any, endogenous protein kinases are susceptible. The typical approach to creating such an "analog-sensitive" allele involves mutating the bulky gatekeeper amino acid residue to glycine or alanine. However, not all kinases can tolerate such a change [3]. By sequence alignment (Supplementary information, Figure S1A), we identified M318 to be the candidate gatekeeper residue for human PINK1. Initially we made a PINK1 M318G mutant, which unfortunately was unstable in mammalian cells (data not shown). An alternative M318A mutant (PINK1 ${ }^{\mathrm{AS}}$ ) was constructed and introduced into PINK1-knockout mouse embryonic fibroblast (MEF) cells that stably express the Venus-Parkin fusion protein. A control wild-type PINK1 $\left(\mathrm{PINK} 1^{\mathrm{WT}}\right.$ ) was used to reconstitute PINK1-null MEF cells in parallel. Loss of mitochondrial membrane potential by decouplers, such as carbonyl cyanide m-chlo- rophenyl hydrazone (CCCP) or valinomycin, is known to prevent PINK1 mitochondrial import and degradation by ParL, resulting in PINK1 accumulation at the outer mitochondrial membrane [1]. Elevated levels of PINK1 promote the translocation of Parkin from the cytosol to the mitochondrion, where Parkin causes ubiquitination and degradation of several proteins localized at the outer mitochondrial membrane to induce mitophagy $[1,2]$. As shown in Figure $1 \mathrm{~A}$, both PINK $1^{\mathrm{WT}}$ and PINK $1^{\mathrm{AS}}$ were effective in inducing Parkin translocation to mitochondria upon treatment with valinomycin. The PP1 analog 1-NA-PP1 inhibited Parkin translocation in cells expressing PINK $1^{\mathrm{AS}}$, but not in cells expressing PINK $1^{\mathrm{WT}}$, demonstrating the specificity of the inhibition (Figure 1A and 1B). PINK1 is known to phosphorylate Parkin in response to mitochondrial depolarization, which leads to a mobility shift of Parkin protein band in immunoblotting assays [4-6] (Figure 1C). In the presence of 1-NA-PP1, the slower migrating Parkin band is completely absent (Figure 1C), suggesting that PINK1 kinase activity is required for Parkin modification. The increase in the expression level of PINK1 upon valinomycin treatment was unaffected by 1-NA-PP1, suggesting that the kinase activity of PINK1 is not required for its increased abundance or its mitochondrial import upon mitochondrial depolarization.

CCCP or valinomycin induced Venus-Parkin translocation to mitochondria within $1 \mathrm{~h}$ with similar kinetics (Supplementary information, Figure S1B). We examined the ability of various PP1 analogs to block PINK1-dependent Parkin translocation in MEF cells expressing $\mathrm{PINK}^{\mathrm{AS}}$ or PINK $1^{\mathrm{WT}}$ (Figure 1D and Supplementary information, Figure S1C). The mitochondrial translocation of Venus-Parkin was quantified $1 \mathrm{~h}$ after CCCP treatment. 1-NA-PP1 showed a nanomolar $\mathrm{IC}_{50}$ value $(165.4 \pm 6.5 \mathrm{nM})$. 1-NM-PP1, 3-MB-PP1 and 2,3-DMBPP1 showed low micromolar $\mathrm{IC}_{50}$ values $(8.3 \pm 0.4 \mu \mathrm{M}$, $10.2 \pm 0.3 \mu \mathrm{M}$ and $20.2 \pm 0.3 \mu \mathrm{M}$, respectively). In contrast, 3-IB-PP1 showed no inhibition when tested at concentrations up to $40 \mu \mathrm{M}$. Importantly, all five bulky PP1 analogs showed no inhibition of wild-type PINK1 at 
A
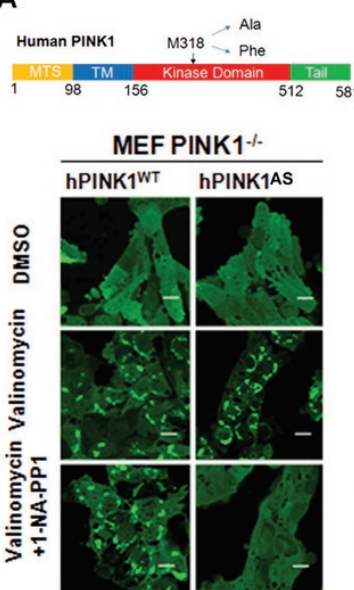

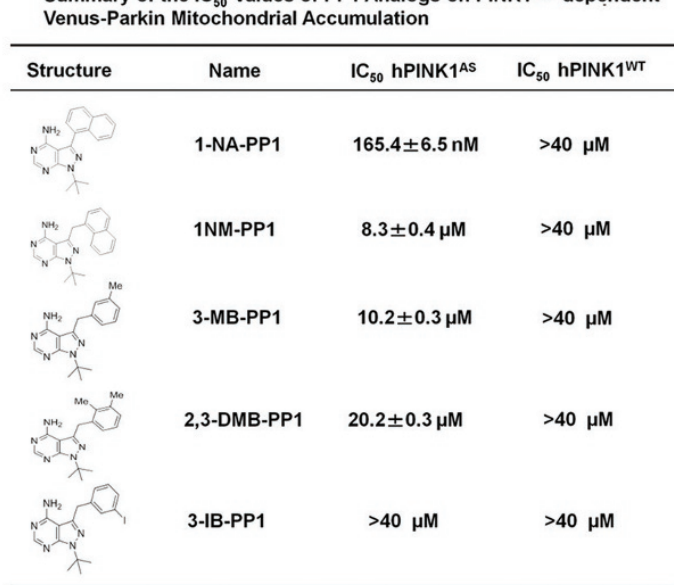

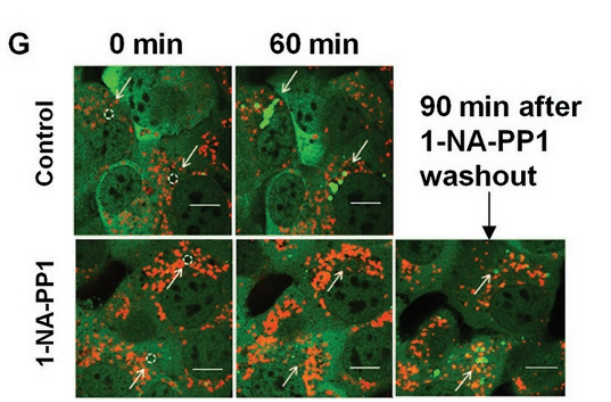

B

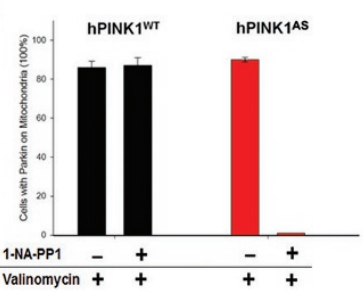

C

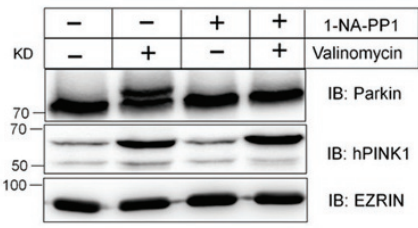

E
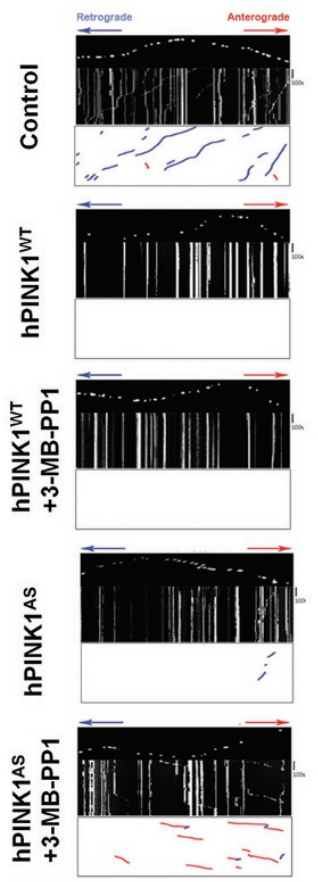

F

CTRL

hPINK1 ${ }^{W T}$

+3-MB-PP1

hPINK1AS

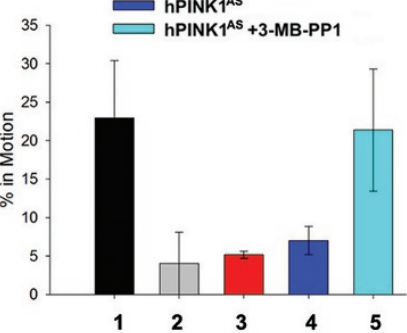

H

Figure 1 Development of the analog-sensitive PINK1 ${ }^{\text {AS }}$ kinase. (A) The putative gatekeeper residue in human PINK1 (upper panel). Valinomycin $(10 \mu \mathrm{M})$ induces mitochondrial translocation of Venus-Parkin in PINK $^{-1}$-Venus-Parkin MEF cells expressing either hPINK $1^{\mathrm{WT}}$ or hPINK1 ${ }^{\mathrm{AS}}$ (lower panel). 1-NA-PP1 $(2 \mu \mathrm{M})$ treatment selectively inhibits hPINK1 ${ }^{\mathrm{AS}}$-induced Parkin translocation. Scale bar, $10 \mu \mathrm{m}$. (B) Quantitation of A. See Supplementary information, Data S1 for details on the calculation. (C) Western blotting of total cell lysate showing that valinomycin $(10 \mu \mathrm{M})$-induced Parkin post-translational modification can be blocked with 1-NA-PP1 (2 $\mu \mathrm{M})$ in PINK $1^{\mathrm{AS}}$-overexpressing MEF cells. (D) IC $_{50}$ values for the inhibitiory effects of PP1 analogs on hPINK1 ${ }^{\mathrm{WT}}$ - or $\mathrm{hPINK} 1^{\mathrm{AS}}$-induced Parkin mitochondrial translocation. (E) Primary mouse hippocampus neurons were transfected with the indicated expression vectors. Forty-eight hours post transfection, 3-MB-PP1 was added for an additional $18 \mathrm{~h}$ prior to imaging. Time-lapse imaging of axonal trafficking of mitochondria was performed. Images were collected every $5 \mathrm{~s}$ for a total of 120 frames. Kymograph analysis shows mitochondrial movement regulated by hPINK $1^{\mathrm{WT}}$ and $\mathrm{hPINK} 1^{\mathrm{AS}}$ with or without 3-MB-PP1 in representative mouse hippocampal axons. (F) Quantitation of the percentage of mobile mitochondria under each condition in $\mathbf{E}$. The $P$ values for each measurement are as follows: 1 vs $2, P=0.079 ; 1$ vs $3, P=0.039 ; 1$ vs $4, P=0.03 ; 1$ vs $5, P=0.446 ; 4$ vs $5, P=0.048$; Student's $t$-test. Error bars represent standard deviation. (G) Photoirradiation (405 nm)-triggered mitochondrial damage in the indicated positions (circles and arrows) induced Venus-Parkin mitochondrial accumulation. In the presence of $2 \mu \mathrm{M}$ 1-NA-PP1, Venus-Parkin accumulation was blocked. Wash-off of 1-NA-PP1 restored Venus-Parkin recruitment. Scale bar, $5 \mu \mathrm{m}$. (H) Temporal dependence of PINK1 ${ }^{\text {AS }}$ inhibition by 1-NA-PP1. If 1-NA-PP1 was added after the commencement of Venus-Parkin translocation, Parkin accumulation on the damaged mitochondria was unaffected. Scale bar, $5 \mu \mathrm{m}$. 
concentrations up to $40 \mu \mathrm{M}$. These results demonstrate the high specificity of PINK $1^{\mathrm{AS}}$ and selectivity of PP1 analogs 1-NA-PP1 and 3-MB-PP1 as orthogonal inhibitors of PINK1 activity.

To further validate the specificity of PINK $1^{\mathrm{AS}}$, we mutated M318 of PINK1 to a bulkier residue Phenylalanine (F) (Figure 1A). PINK1 $1^{\mathrm{WT}}$, PINK1 $1^{\mathrm{AS}}$, and PINK $1^{\mathrm{M} 318 \mathrm{~F}}$ all supported CCCP-induced Venus-Parkin translocation, indicating that they all exhibit functional kinase activity (Supplementary information, Figure S1D-S1F). EC $\mathrm{E}_{50}$ values for the effect of CCCP on Parkin mitochondrial translocation in HeLa and MEF cells are summarized in Supplementary information, Figure S1G-S1H. 3-MBPP1 inhibited the activity of PINK1 ${ }^{\text {AS }}$, but not that of PINK $1^{\mathrm{WT}}$ or PINK $1^{\mathrm{M} 318 \mathrm{~F}}$. These results further confirm that these PP1 analogs exclusively inhibit PINK $1^{\mathrm{AS}}$ activity.

We next attempted to demonstrate the direct inhibition of PINK $1^{\mathrm{AS}}$ by PP1 analogs using in vitro kinase assays, but failed to detect robust kinase activity of recombinant human PINK1. Since an insect orthologue of PINK1 from Tribolium castaneum (beetle; TcPINK1) was shown to be catalytically active [4], we substituted TcPINK1 M294 which is equivalent to M318 of human PINK1 with Alanine. Recombinant myelin basic protein (MBP)-tagged wild-type TcPINK1 and the mutant MBP-TcPINK $1^{\mathrm{M} 294 \mathrm{~A}}$ were purified and tested for their kinase activity in the presence or absence of 3-MB-PP1 or 1-NA-PP1. Both MBP-TcPINK1 and MBP-TcPINK$1^{\mathrm{M} 294 \mathrm{~A}}$ can phosphorylate recombinant Parkin1-108 in vitro. No inhibition of MBP-TcPINK $1^{\text {WT }}$ was observed by either 1-NA-PP1 or 3-MB-PP1 in the dose range tested (Supplementary information, Figure S1I). In contrast, both 1-NA-PP1 and 3-MB-PP1 inhibited MBP-TcPINK$1^{\mathrm{M} 294 \mathrm{~A}}$ activity, albeit with $\mathrm{IC}_{50}$ values higher than those measured with human PINK $1^{\mathrm{AS}}$ in cell culture models. The reason for this discrepancy is likely due to sequence differences in the ATP-binding pocket between human and beetle PINK1, where these inhibitors bind. Nevertheless, the fact that PP1 analogs did not inhibit TcPINK $1^{\text {WT }}$ but inhibited the TcPINK $1^{\mathrm{M} 294 \mathrm{~A}}$ mutant further substantiates that this methionine residue is the gatekeeper residue for PINK1.

As we expressed human PINK1 in PINK1-null MEF cells, it is difficult to compare the expression level of hPINK1 to the level of endogenous mouse PINK1 because the PINK1 antibody (Novus) has different reactivity to human and mouse PINK1. Nevertheless, the response of MEF cells reconstituted with human PINK1 to CCCP treatment is comparable to that of HeLa cells (Supplementary information, Figure S1G and S1H), suggesting that the activity of human PINK1 in mediating
Parkin translocation in MEF cells is similar to that in HeLa cells. Hence, the MEF system can capture the essential features of the PINK1-Parkin pathway.

A key advantage of employing a pharmacological tool to perturb gene function versus the traditional knockout or knockdown approach lies in its spatiotemporal resolution and separation of short-term versus long-term effects. Overexpression of PINK1 has been shown to activate the PINK1-Parkin pathway and decrease the mobility of axonal mitochondria in neurons [7]. To verify the applicability of the PINK $1^{\mathrm{AS}}$ in mitochondrial mobility studies, we expressed PINK $1^{\mathrm{WT}}$ and PINK $1^{\mathrm{AS}}$ in primary mouse neurons. We examined the mobility of axonal mitochondria in mouse hippocampal neurons stained with mitoTracker to monitor mitochondrial movement in the presence or absence of 3-MB-PP1, which has previously been demonstrated to be suitable for studying analog-sensitive kinases in primary neurons [8]. While expression of both PINK $1^{\mathrm{WT}}$ and PINK $1^{\mathrm{AS}}$ indeed restricted the mitochondrial movement, treatment with 3-MB-PP1 for $18 \mathrm{~h}$ rescued the mobility in neurons expressing PINK1 ${ }^{\mathrm{AS}}$, but not in neurons expressing

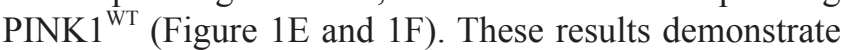
that this tool is valuable for studying PINK1-dependent mitochondrial mobility events and allows relatively rapid inactivation of PINK1 kinase activity in primary neurons, which otherwise would not be feasible with knockdown or knockout approach.

The availability of PINK $1^{\mathrm{AS}}$ enabled us to test the requirement of PINK1 in initiation and maintenance of mitophagy in a spatiotemporal manner. Treatment with CCCP or valinomycin causes global mitochondrial damage in cells. Damage of an individual mitochondrion by $488 \mathrm{~nm}$ laser has been shown to trigger mitophagic responses locally [9]. To test whether PINK1 kinase activity is required for initiation of mitophagy upon laser irradiation, selected mitochondria in MEF cells expressing PINK $1^{\mathrm{AS}}$ and Venus-Parkin were exposed to $488 \mathrm{~nm}$ or $405 \mathrm{~nm}$ laser illumination. This treatment caused rapid depolarization, as measured by TMRE dye that loses fluorescence upon mitochondrial depolarization. Indeed, local laser exposure rapidly induced Parkin accumulation on irradiated mitochondria (Figure 1G). Extra Parkin foci appearing outside irradiated areas occasionally are either due to a bystander effect described previously [9] or to mitochondria that were photodamaged beneath the imaging plane and then reappeared in the focal plane. Incubation of cells with 1-NA-PP1 prior to laser exposure abrogated local Parkin accumulation. Upon washout of 1-NA-PP1, Parkin accumulated on damaged mitochondria (Figure 1G). These results suggest that PINK1 is required for initiating Parkin translocation to damaged 
mitochondria. To test whether PINK1 is also required for sustaining Parkin accumulation on damaged mitochondria, we added 1-NA-PP1 20 min after laser exposure when Parkin translocation had already occurred (Figure $1 \mathrm{H}$, middle panel). Parkin accumulation on mitochondria was sustained even in the presence of 1-NA-PP1, suggesting that maintenance of Parkin at mitochondria does not require PINK1 activity.

We then performed a similar experiment in which we inhibited PINK $1^{\mathrm{AS}}$ activity after CCCP treatment, which is known to trigger clustering of mitochondria [1]. As expected, CCCP treatment for $6 \mathrm{~h}$ led to clustering of mitochondria (Supplementary information, Figure S1J and S1K, RFP channel). When CCCP was added together with 1-NA-PP1, the effect of CCCP was abrogated. When we added CCCP $1 \mathrm{~h}$ prior to the incubation with 1-NA-PP1, CCCP-induced mitochondrial clustering was not reversed by 1-NA-PP1. These results strongly suggest that PINK1 can initiate Parkin translocation to the mitochondria, but is dispensable for the persistent presence of Parkin on damaged mitochondria. This could be a result of a feed-forward loop triggered by PINK1, as is the case of ubiquitin phosphorylation or autocatalytic ubiquitination of Parkin, creating a bistable system (Supplementary information, Figure S1L) [6, 10-13].

The PINK1-Parkin pathway has also been implicated in modulating mitochondrial fission/fusion dynamics by inducing ubiquitination of Drp1 or Mitofusins [1, 14, 15]. Indeed, in MEF cells expressing hPINK $1^{\mathrm{AS}}$, mitochondria are predominantly fragmented (Supplementary information, Figure S1M). Upon treatment with 1-NAPP1, many MEF cells showed gradual increase in mitochondrial fusion frequency with mitochondria in over $30 \%$ of MEF cells displaying more tubular morphology after $8 \mathrm{~h}$. This is consistent with the notion that PINK1 inhibition facilitates mitochondrial fusion.

In summary, we have successfully engineered an analog-sensitive PINK1 kinase that can be specifically inhibited by orthogonal small molecules. The pharmacological tool described here should aid future research on the role of PINK1-Parkin pathway in areas including but not limited to the regulation of mitochondrial protection, mitophagy, mitochondrial mobility, mitochondrial fission/fusion, cellular metabolism, and apoptosis. Despite a strong association between mitochondrial dysfunction and the PINK1-Parkin pathway in Parkinson's disease, it remains unclear how endogenous PINK1 and Parkin contribute to mitophagy or mitochondrial dysfunction in neurons [2]. Conceivably, the PINK $1^{\mathrm{AS}}$ allele can be introduced into iPS or ES cells via knockin techniques to produce animals with a PINK1 that can be pharmacologically perturbed to evaluate both short- and long-term effects of inhibiting this pathway on neurodegenerative and perhaps other diseases.

\section{Acknowledgments}

We thank Dr Kevan Shokat for providing critical reagents, Drs Kevin Dean, Yan Qin and Genevieve Park for technical assistance, Drs James Goodrich and Sabrina Spencer for their critical reading of the manuscript, Drs Natalie Ahn, Amy Palmer, Ding Xue, Hubert Yin and members of the Liu laboratory for discussion on the manuscript and Drs Muqit, Walden, Miyoshi and Spencer for expression vectors. This work was supported by grants from the Butcher Award, Cancer League of Colorado, and National Institutes of Health (CA107098 to XL and R01NS041779 to JS). EB was supported by a predoctoral training grant from NIGMS (T32GM08759). The ImageXpress was supported by an NCRR grant S10 RR026680 from NIH.

Conggang Zhang ${ }^{1}$, Schuyler Lee ${ }^{1}$, Yinghua Peng ${ }^{1}$, Eric Bunker ${ }^{1}$, Chong Shen ${ }^{2}$, Emilie Giaime ${ }^{3}$, Jie Shen ${ }^{3}$, Jingshi Shen ${ }^{2}$, Zongyao Zhou ${ }^{1}$, Xuedong Liu ${ }^{1}$

\begin{abstract}
${ }^{I}$ Department of Chemistry and Biochemistry, Jennie Smoly Caruthers Biotechnology Building (JSCBB), ${ }^{2}$ Department of MCD-Biology, University of Colorado-Boulder, Boulder, 3415 Colorado Ave, CO 80303, USA; ${ }^{3}$ Center for Neurologic Diseases, Brigham and Women's Hospital, Program in Neuroscience, Harvard Medical School, New Research Building, Rm 636E, 77 Avenue Louis Pasteur, Boston, MA 02115, USA

Correspondence: Xuedong Liu

Tel: 001-303-735-6161; Fax: 001-303-735-6161

E-mail: Xuedong.Liu@colorado.edu
\end{abstract}

\section{References}

1 Youle RJ, Narendra DP. Nat Rev Mol Cell Biol 2011; 12:9-14.

2 Sugiura A, McLelland GL, Fon EA, et al. EMBO J 2014; 33:21422156.

3 Dar AC, Shokat KM. Annu Rev Biochem 2011; 80:769-795.

4 Kondapalli C, Kazlauskaite A, Zhang N, et al. Open Biol 2012; 2:120080.

5 Hertz NT, Berthet A, Sos ML, et al. Cell 2013; 154:737-747.

6 Zhang C, Lee S, Peng Y, et al. Curr Biol 2014; 24:1854-1865.

7 Wang X, Winter D, Ashrafi G, et al. Cell 2011; 147:893-906.

8 Chen M, Maloney JA, Kallop DY, et al. J Neurosci 2012; 32:1343913453.

9 Kim I, Lemasters JJ. Antioxid Redox Signal 2011; 14:1919-1928.

10 Zheng X, Hunter T. Cell Res 2013; 23:886-897.

11 Kane LA, Lazarou M, Fogel AI, et al. J Cell Biol 2014; 205:143-153.

12 Kazlauskaite A, Kondapalli C, Gourlay R, et al. Biochem J 2014; 460:127-139.

13 Koyano F, Okatsu K, Kosako H, et al. Nature 2014; 510:162-166.

14 Yang Y, Ouyang Y, Yang L, et al. Proc Natl Acad Sci USA 2008; 105:7070-7075.

15 Wang H, Song P, Du L, et al. J Biol Chem 2011; 286:11649-11658.

(Supplementary information is linked to the online version of the paper on the Cell Research website.) 